\title{
Retraction of the Paper
}

Title: Using Semantic Clustering for Detecting Bengali Multiword Expressions Author: Tanmoy Chakraborty

Published in Informatica, Vol 38, No 2, 2014 http://www.informatica.si/index.php/informatica/article/view/690

Due to:

Tanmoy Chakraborty, October 19, 2019

Dear Editor,

I would like to retract the paper, titled "USING SEMANTIC CLUSTERING FOR DETECTING BENGALI MULTIWORD EXPRESSIONS", authored by Tanmoy Chakraborty, published in Informatica (Vol 38, No 2, 2014). The URL of the paper is http://www.informatica.si/index.php/informatica/article/view/690. The reason is that the dataset that we used in this paper are not publicly available and copyright protected. We collected the dataset from https://rabindra-rachanabali.nltr.org/. We recently realized that the content of this site is owned by SNLTR. Therefore, a prior permission is needed before using the content of the website. Moreover, the paper does not align to my current mainstream research.

Thank you very much for your understanding.

Best regards,

Tanmoy Chakraborty 
